\section{(G) bretrátionz}

ON

\section{THE PRODUCTION OF MENINGOCOCCUS ANTI-ENDOTOXIN.}

BY

M. H. GORDON, C.M.G., M.D.,

Temporary Honorary Liedtenant-Colonel R.A.M.C.

(From the Central C.S.F. Laboratory, London.)

In a paper published in the British Medical Journal of January $26 \mathrm{th}, 1918$, attention was drawn to the fact that the growth from young cultures of virulent specimens of the meningococcus, when killed by ether, dried in vacuo and powdered, was toxic to mice intraperitoneally in a dose varying from 2 to $10 \mathrm{mg}$. By carefully and thoroughly grinding 0.1 gram of this powder in an agate mortar, after the method of endotoxin extraction described by Besredka, by slowly adding $5 \mathrm{c.cm}$. of distilled water, and then centrifuging out the heavier particles, an opaque watery extract was obtained that contained enough of the intracellular poison of the meningococcus to kill mice intraperitoneally in a dose varying from 0.1 to $0.2 \mathrm{c.cm}$. Considerable 'differences were found in the capacity of various specimens of antimeningococcus serum to neutralize the endotoxin obtained in this manner from each of the two commonest types of the meningococcus. Some therapeutic serums, in spite of a high content of opsonin and agglutinin, proved to be deficient in antiendotoxin. Two samples of serum, however, emerged successfully from the test, and were found to neutralize I minimal lethal dose of the endotoxin of both types I and II of the meningococcus with $0.5 \mathrm{c.cm}$. of serum. The first was a sample from a batch that had given far better results therapeutically in military cases than any other serum employed up to that date. At the time that the second serum was submitted to the test for antiendotoxin it had not yet been tried clinically, but in view of its evident toxin-neutralizing qualities this serum was strongly recommended for therapeutic use during the earlier mouths of the present year, especially in cases infected by Type I meningococcus, for which it contained more anti-endotoxin than for Type II. The results have been exceedingly satisfactory.

From this experience, and also from the fact that specimens of multivalent serum that proved to have less therapeutic value than the two serums in question failed without exception to neutralize 1 minimal lethal dose of the endotoxin of the two commonest types of the meningococcus with $0.5 \mathrm{c.cm}$. of the serum, there is ground for believing that in the case of antimeningococcus serum the highest clinical potency is not obtained unless the serum has a definite amount of anti-endotoxin. Nor is an excellent reason for this far to seek, since the pathogenicity of the meningococcus is admittedly due to its endotoxin. Meningococcus antiendotoxin is by no means easy to obtain, since at pre sent only occasional batches of antimeningococcus serum are found to contain it in any quantity. Nevertheles it would seem to be very desirable that antimeningococcus serum should possess as high a value as possible in regard to anti-endotoxin. But before serum of this quality can be prepared on a scale large enough to reduce materially the present aggregate mortality from cerebro-spinal fever further information is needed concerning the production of meningococcus anti-endotoxin. For this reason an in. vestigation was begun for the purpose of defining the value of various preparations of the meningococcus for stimulating the production of anti-endotoxin in the rabbit.

\section{Procedure.}

Young rabbits of 1,000 to 2,000 grams weight were used and all the injections were made intravenously. The antigens compared have been (1) the raw coccus suspended in saline; (2) the same coccus after being sensitized by leaving it all night in contact with a good specimen of antimeningococcus serum, which is drawn off next morning and the agglutinated cocci resuspended in saline; (3) the raw coccus autolyzed by leaving it for three days at $37^{\circ} \mathrm{C}$.; (4) the raw coccus softened and partially dissolved by suspending it in $\mathrm{N} / 40 \mathrm{NaOH}$; (5) a standurd suspension
(0.1 gram powder to 5 c.cm. water) of the dried coccus ground up and suspended in distilled water: (6) a suspension of the dried coccus of similar strength but with the endotoxin inactivated by leaving it in the incubator overnight in contact with $\mathrm{N} / 1 \mathrm{NaOH}$ subsequently reduced to $N / 40$. All of the above antigens were preserved by the addition of 0.5 per cent. of phenol.

Samples of serum have been tested for anti-endotoxin in the following way. At first a standard extract of the dried coccus was used, but later, with multiplication of the tests, it was found impossible to spare the time necessary for making these extracts, and so recourse has been had mainly to suspensions of 0.1 gram of the dried and powdered coccus ground up for about ten minutes in $5 \mathrm{c.cm}$. of distilled water and well shaken, but not centrifuged. A's a preservative, 10 per cent. of ether is added, phenol being avoided because it is toxic for the mice. Such suspensions vary somewhat in toxicity according to the thoroughness of the grinding. I have preferred to use watery suspen sions as a rule, but sometimes the results may be rendered more uniformly fatal in the controls by the addition of $\mathrm{N} / 40 \mathrm{NaOH}$ to the suspension by grinding up 0.1 gram of the powder in $2.5 \mathrm{c} . \mathrm{cm}$. of distilled water, and then adding $2.5 \mathrm{c.cm}$. of $\mathrm{N} / 20 \mathrm{NaOH}$. The objection to $\mathrm{NaOH}$ is that while it tends to dissolve the dried coccus it also tends, especially if too concentrated, to destroy the toxin. Before using it for the present purpose the ninimal lethal dose of $\mathrm{NaOH}$ for the mouse intraperitoneally was determined, and found to be $0.5 \mathrm{c.cm}$. of a N/10 solution, or $2 \mathrm{mg}$. In testing serums the minimal lethad dose of the endotoxin - generally from 0.1 to 0.2 of the standard suspension-is measured out into a series of watch-glasses enclosed in Petri dishes; $0.5 \mathrm{c.cm}$. of serum is then added to each, and the Petri dish transferred to the $37^{\circ} \mathrm{C}$. incubator for thirty minutes, at the end of which time the contents of the watch-glasses are injected intraperitoneally into mice, preferably of between 12 and 20 grams weight. The mice are kept under observation for two to three days. With potent toxin, of course, the life or death of the mouse is the criterion, but with weaker toxin the presence or absence of illness is a good index to the antitoxic value of the serum. To this end the mice should be individually inspected, especially on the morning after injection, and a note made with regard to their appetite, mobility, and particularly the question as to whether their eyes are open or not, for mice affected by meningococcus endotoxin tend to keep their eyes shat. When carrying out tests on a large number of samples of serum (for example, twenty or more at a time) I have found it convenient to use two specimens of the same endotoxin. In the first place, $0.5 \mathrm{c.cm}$. amounts of the samples are put up against a weaker toxin in order to separate those with any neutralizing powers from the rest; the serums thus selected are then retested against more potent toxin and their capacity to save the life of a mouse from its effects ascertained. If necessary, serums that neutralize in $0.5 \mathrm{c.cm}$. can then be tested in the same way in smaller amounts.

Four series of observations have been carried out so far, and the results may be summarized as follows:

SERIES I.

In December, 1917; eleven rabbits were prepared with Type I meningococcus, strain Howes, as follows: Two with the ra coccus, two with the same after sensitization, and two with the coccus after autolysis for three days at $37^{\circ} \mathrm{C}$. The remaining five rabbits received the watery extract of the dried coccus. Throughout the experiment the dose was raised at each injection and the animals were frequently weighed.

T'est 1.-On January 7th, 1918, the twenty-first day of the experiment and three dars after the fourth dose, samples experiment and three days after the fourth dose, samples
of blood were taken from all the rabbits and the serum tested for anti-endotoxin. Result: Five rabbits positive and six for anti-endotoxin. Result : Five rabbits positive and six negative. The positive rabbits were: Both the animals inoculated with the sensitized coccus, both the autolyzed
coccus rabbits, and one of the four rabbits prepared with
the extract of the dried coccus. The injections were continued, the dose being raised further, and on February 4th, five days after the sixth dose, the test was repeater. 'Two of the rabbits had died-namely, one of those prepared with the sensitized coccus and one of those prepared with the extract, so that nine rabbits were left.

'T'est 2.-Four of the rabbits were positive and five negative. The positive animals were the rabbit prepared with the sensitized coccus, both those prepared with the sutolysate and one of the rabbits prepared with the extract. The in jections were continued prepared wing the extract. The in jections were continued, the dose being raised further, and on made. Five of the rabbits now survived-namely, one of those 
prepared with the sensitized coccus, both those prepared with he autolysate, and two of those prepared with the extract.

I'est 3.- Only two of these five rabbits showed anti-endotoxin -namely, one of the rabbits prepared with the autolysate, and one of those prepared with the extract.

\section{SERIES II.}

While the preceding experiment was in progress another one was started in which four rabbits were prepared with equal doses of the same coccus (Type I, Howes) as follows: (1) Coccus raw, (2) coccus sensitized, (3) coccus dried, (4) coccus dried and sensitized. On the twenty-first day of the experiment, six days after the fourth dose (4,000 million cocci) had been given, the serum of these rabbits was tested for anti-endotoxin, with result that the animal prepared with the raw coccus and that prepared with the dry coccus after sensitization were both positive, the other two negative. All of the rabbits now received three further injections at intervals of a week as follows: 10,000 million, 20,000 million, and 25,000 million, and six days after the last injection another test was made, with the result that the serum of all the rabbits was now found to be negative.

Though these two experiments failed to settle the question as to which is the best antigen for evoling the production of anti-endotoxin, they nevertheless brought out the important point that for the production of antiendotoxin to Type $I$ it is very necessary not to raise the dose too high. So far from increasing the yield of antiendotoxin, super-immunization may cause it to disappear.

\section{SERIES III.}

At the time that Series I was started a similar group of eleven rabbits was prepared in the same way with Type II meningococcus, strain Morgan. These rabbits received doses of the same size as those given to the rabbits in Series I, the intervals were similar and the dose was raised in much the same way. were similar and the dose was raised in much the same way.
The first dose was 500 millions and the last 10,000 millions. In The first dose was 500 millions and the last 10,000 millions. In rabbits died during immunization-an experience that confirms a previous observation, namely, that Type I is more toxic than II to laboratory animals. No examination was made of the antitoxic value of the serum of these rabbits until they had been under preparation for a period of three months, during which time they received nine injections. Ten days after the last injection a sample of blood was taken from each animal and tested for anti-endotoxin (Type II) with the following result :

\begin{tabular}{|c|c|c|c|c|c|c|c|c|c|}
\hline \multirow{2}{*}{ No. } & \multirow{2}{*}{ Antigen. } & \multicolumn{3}{|c|}{ Serum. } & \multirow{2}{*}{ No. } & \multirow{2}{*}{ Antigen. } & \multicolumn{3}{|c|}{ Serum. } \\
\hline & & 0.5 & 0.25 & 0.1 & & & 0.5 & 0.25 & 01 \\
\hline 1 & Raw coccus & - & & & 7 & Dried coccus ... & + & + & + \\
\hline 2 & Raw coccus $\quad \ldots$ & + & + & + & 8 & Dried coccus ... & + & + & - \\
\hline 3 & Sensitized coccus & + & - & & 9 & Dried coccus ... & + & + & - \\
\hline 4 & Sensitized coccus & + & - & & 10 & Dried coccus ... & + & + & - \\
\hline 5 & Autolyzed coccus & - & & & 11 & Dried coccus ... & - & & \\
\hline 6 & Autolyzed coccus & - & & & & & & & \\
\hline
\end{tabular}

The sign + indicates that the serum neutralized 1 minimal lethal dose of endotoxin.

It would seem that anti-endotoxin is more readily elaborated by the rabbit to Type II meningococcus than to 'Type I. The sensitized coccus and the suspension of dried coccus would appear to be more consistently successful than the other antigens here tried.

\section{SERIES IV.}

The next step was to resume comparison of various antigens of Type I, but with precautions against overdosage. To this end the present experiment was performed in duplicate, one set of rabbits receiving the same dose throughout, the dose being very slowly increased in the other. An interval of about fortyeight hours was allowed to elapse between the injections, and each rabbit received altogether ten doses.

'The antigens compared were: (1) A suspension of the raw coccus in saline; (2) the same coccus after being sensitized; (3) the same coccus after being partially dissolved in N/40 NaOH; (4) A standard suspension of the dried coccus; (5) a suspension of the dried coccus of strength similar to the last, but with the toxin inactivated by leaving it in contact overnight in the incubator at $37^{\circ} \mathrm{C}$., with $\mathrm{N}_{1}^{\prime} \mathrm{NaOH}$, subsequently reduced to incubat

Particulars of the mode of preparation of these rabbits are seen in the following table, which shows details of the treatment given to the five rabbits in which the dose was raised. In the five other rabbits the dose here given on the first day was repeated on all the subsecutent nine occasions without being raised.
Table showing Details of Treatment in Five Rabbits in which the Dose was raised.

The figures for Nos. 1, 2, and 3 represent millions of cocci; those for Nos. 4 and 5, cubic centimetres of standard suspension.)

\begin{tabular}{|c|c|c|c|c|c|c|c|c|c|c|c|}
\hline No. & Antigen. & Day: & 4 & 6 & 8 & 10 & 12 & 14 & 17 & 19 & 21 \\
\hline 1 & Raw coccus ... & 500 & 500 & 600 & 600 & 700 & 700 & 800 & 800 & 900 & 1000 \\
\hline 2 & Sensitized coccus ... & 500 & 500 & 600 & 600 & 700 & 700 & 800 & 800 & 900 & 1000 \\
\hline 3 & $\begin{array}{l}\text { Coccus dissolved in } \\
\mathrm{NaOH}\end{array}$ & 500 & 500 & 600 & 600 & 700 & 700 & 800 & 800 & 900 & 1000 \\
\hline 4 & Dried coccus ... & 0.05 & 0.05 & 0.06 & 0.06 & 0.07 & $0.0^{\circ}$ & 0.08 & 0.08 & 0.09 & 0.1 \\
\hline 5 & $\begin{array}{l}\text { Dried coccus dis- } \\
\text { solved in } \mathrm{NaOH}\end{array}$ & 0.05 & 0.05 & 0.0 & 0.08 & $0.0^{\circ}$ & $0.0^{\prime}$ & 0.08 & 0.08 & 009 & 0.1 \\
\hline
\end{tabular}

Two specimens of the endotoxin of the meningococcus used in this experiment were employed for testing the serum of the rabbits for anti-endotoxin. The first toxin was a strong one, since 0.1 c.cm. of it invariably killed the controls. The second toxin, on the other hand, while it produced a severe illness in the dose given- 0.1 c.cm.- did not usually kill. While less stringent as a test for antiendotoxin than the first toxin, therefore, it was more deli. cate. For this reason, in the tests for anti-endotoxin made after the rabbits had received the fifth and tenth injections, the serum of all ten rabbits was first of all examined against the weak toxin, and the serums that neutralized this were then tested against the stronger toxin.

\section{Results.}

\section{First I'est.}

On the third day of the experiment, and forty-eight hours after the first dose, $0.5 \mathrm{c.cm}$. of serum from each rabbit was mixed with $0.1 \mathrm{c.cm}$. of the strong toxin, and after thirty minutes at $37^{\circ} \mathrm{C}$. injected intraperitoneally into a mouse. All the mice were dead within forty-eight hours, and cultures made from their hearts' blood were, as usual, sterile. At this stage, therefore, none of the rabbits gave any evidence of anti-endotoxin.

\section{Second Test.}

On the eleventh day of the experiment, twenty-four hours after the rabbits had received their fifth injection, the serum of each rabbit was tested against 0.1 c.cm. of the weak toxin. Six of the serums failed to neutralize it and four inactivated it, the mice remaining unaffected. The rabbits providing the successful serum had received the following antigens:

1. The raw coccus, dose stationary.

2. The dried coccus, dose stationary.

3. The dried coccus, dose raised.

4. The sensitized coccus, dose raised.

The serums from these four rabbits were now tested against the strong toxin, with the result that two failed to save the mice and two succeeded. The successful serums were those from the rabbits that had received rising doses of the dried coccus and of the sensitized coccus re spectively; $0.25 \mathrm{c.cm}$. of each serum was then put up against the weak toxin, but failed to neutralize it.

\section{Third Test.}

On the twenty-first day of the experiment, twenty-four hours after the tenth dose, the serum of all the rabbits was once more tested against the weak toxin. Six of the serums failed to neutralize it and four succeeded. The four successful serums came from the rabbits prepared with :

1. The dried coccus, dose stationary.

2. The dried coccus, dose raised.

3. The sensitized coccus, dose raised.

4. The raw coccus, dose raised.

On testing these four serums against the strong toxin, the serum from the rabbit with the raw coccus failed, the rest succeeded in saving the mice. The three successful serums were now tested against the strong toxin, but only 0.25 c.cm. of serum was used instead of 0.5 c.cm. as before. The serum from the rabbit prepared with the sensitized coccus failed, the other two succeeded. Both the successful serums were then tested in $0.1 \mathrm{c.cm}$. amounts against the weak toxin, with result that the serum from the rabbit that had received rising doses of the dried coccus 
neutralized it, while that from the rabbit that had received the stationary dose of this same antigen failed to do so.

\section{Fourth Test.}

On the third day after the tenth dose the serum of all the rabbits was tested against the weak toxin, but none of the rabbits previously negative was found to be other than negative again. The serum of the rabbits prepared with the following antigens neutralized the toxin:

$$
\begin{aligned}
& \text { 1. The dried coccus, dose stationary. } \\
& \text { 2. The dried coccus, dose raised. } \\
& \text { 3. The sensitized coccus, dose raised. } \\
& \text { 4. The raw coccus, dose raised. }
\end{aligned}
$$

On testing these serums against the strong toxin, two saved the mice and two failed to do so. The successful serums came from the rabbits prepared respectively with the sensitized coccus and with the dried coccus, both in rising doses. On proceeding to test $0.25 \mathrm{c.cm}$. of each serum against the strong toxin, neither of them succeeded in saving the mice. Anti-endotoxin, therefore, was far more abundant in the serum of both these rabbits twentyfour hours after receiving the last dose than two days later.

Fifth Test.

On the fifth day after the tenth dose the serums of the two rabbits positive in the last test were re-examined and both found to be negative in $0.5 \mathrm{c.cm}$.

\section{Summary.}

1. Multivalent antimeningococcus serum that has given the best results in military cases has been found to be differentiated from serum of less therapeutic value by the fact that the former contains a demonstrable quantity of anti-endotoxins to each of the two commonest types of the meningococcus.

2. Anti-endotoxin is elaborated by the rabbit to both the commonest types of the meningococcus.

3. In order to obtain anti-endotoxin, especially in the case of Type I meningococcus, it is absolutely essential to avoid over-dosage.

4. The antigens that appear to give best results in the rabbit are neither the raw coccus nor autolysates, but (1) a suspension of the dried coccus, and (2) the sensitized raw coccus.

\section{THE VALUE OF $X$ RAYS IN THE TREATMENT OF MALIGNANT DISEASE OF THE BREAST.*}

By CLAUDE SABERTON, M.D., HARROGATE.

THE subject matter of this paper is not new, but having had ten years' personal experience in the $x$-ray treatment of malignant disease, I propose to discuss its value in malignant disease of the breast, for the $x$-ray treatment of malignant disease in general is too large a subject for a single paper. The cases we are called upon to treat fall into three main groups: (1) Those sent for prophylactic treatment following operation, or (2) for treatment of recurrences, and (3) inoperable cases.

We will consider these groups seriatim.

\section{Prophylactic Treatient.}

Patients for prophylactic treatment should be sent to the radiologist as soon as possible after operation. It is essential to administer large doses of $x$ rays, and the rays are measured after filtration through 2 or $3 \mathrm{~mm}$. of aluminium. The scar area, axilla, supraclavicular and post-scapular areas should all be treated. With careful technique these doses can be repeated to the same skin areas once a fortnight. This method of cross fire enables a large quantity of rays to reach the mediastinum. The radiation is greably facilitated by the introduction of the Coolidge tube. In ordinary cases the first course of treatment lasts about three months. A second shorter course is given after two or three months, and subsequent radiations are administered at gradually lengthening intervals. The patient is kept under observation for two or three years following the date of the operation. During this

* Read before the Yorkshire Branch of the British Medical Association at Harrogate. July, 1918 . time it is important for the pliysician in charge of the case to administer blood tonics, such as iron, and give advice as to general habits of life, so that the patient's resisting powers may be built up. In some cases the administration of thyroid extract is advisable. Blood counts at intervals are a helpful guide, and last, but not least, I must lay stress on the great help and importance of optimism. The patient needs all her powers and must be cheered and encouraged in the fight.

\section{Results.}

Local scar recurrences are rare, but if nodules appear they can with rare exceptions be controlled. I have seen one or two cases in which there was a rapid spread of cutaneous nodules all over the body, probably due to a general infection of the lymphatic system of the skin. When this occurs patients usually succumb rapidly.

Slight enlargement of glands and thickening belind and above the clavicle are fairly common. We are able to prevent gross, enlargement of these supraclavicular deposits, and they frequently disappear under vigorous raying. They never break down or ulcerate; I think they are sometimes possibly more of an inflammatory nature than actually malignant, and are perhaps due to some slight septic infection of the wound area. The question arises whether these supraclavicular enlargements are recurrences following the operation, or due to inability to remove in fected glands at the time of operation owing to their situation behind the clavicle. Certainly supraclavicular enlargements are much more frequent than axillary deposits. I have never seen ulceration from the breaking down of secondary growths in cases that have had the benefit of an operation followed by intensive $x$-ray therapy. On the other hand, large foul fungating and ulcerated masses are frequently seen in patients not subjected to radiation.

We are frequently disappointed when all appears to be well, and there is no evidence of any local recurrence or mediastinal deposit, by patients complaining of lumbago or sciatica. It is curious how often one meets with recur. rences in the lumbar spine; unfortunately at present all one can hope to do in these cases is to relieve or alleviate pain.

Swelling of the arm from lymphatic obstruction is occasionally a troublesome and distressing sequel of operations, and is most common where it has been necessary to remove a large area of skin. Owing to fixation of the tight cutaneous tissues to the thoracic wall, and the forma. tion of taut bands of scar tissue running along the anterior fold of the axilla, the patient is from the first unable to abduct the arm. This condition materially interferes with the efficient radiation of the armpit. Usually the lymphatic obstruction of the arm gets progressively worse, and the limb sometimes reaches an enormous size. This com. plication is not so frequent as it was years ago, probably owing to different operative technique, and I have noticed it to be much less common in those cases in which the surgeon has carried the skin incision along the anterior fold of the axilla and not into the axilla.

The general conclusions arrived at from treatment of cases in this group are as follows:

1. The patients are less likely to have a recurrence of the disease than cases not so treated.

2. Local recurrence in the scar area is rare.

3. Supraclavicular deposits do occur, but can as a rule be controlled. These deposits never break down or ulcerate.

4. Mediastinal recurrence appears to be less frequent.

5. The general health of the patient is improved.

In view of these facts every case of scirrhus mammae should be subjected to efficient $x$ :ray therapy as soon as possible after operation.

In slow-growing atrophic scirrhus mammae met with in patients about 50 year's of age, cases with perhaps a history of ten to fifteen or more years, it is my experience that operation is not as a general rule advisable even though the growth may be showing signs of activity. I have seen a number of these cases after operation in which the general health has broken down, metastases have almost immediately occurred, and the patient has rapidly gone downhill. These results may be due to the removal of the old natural fibrous wall built around the tumour, and the lowered resistance of the patient following the operation. Unless the surgeon decides to employ 